\title{
The importance of urotensin II level in the diagnosis of acute mesenteric ischemia
}

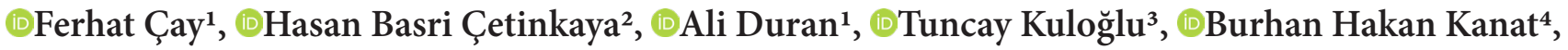 \\ ๑Bilal Üstündağ ${ }^{5}$ \\ ${ }^{1}$ Balıkesir University School of Medicine, Department of General Surgery, Balıkesir, Turkey \\ ${ }^{2}$ Balıkesir University School of Medicine, Department of Emergency Medicine, Balıkesir, Turkey \\ ${ }^{3}$ Firat University School of Medicine, Department of Histology and Embryology, Elazı̆̆, Turkey \\ ${ }^{4}$ Turgut Özal University School of Medicine, Department of General Surgery, Malatya, Turkey \\ ${ }^{5}$ Firat University School of Medicine, Department of Medical Biochemistry, Elazığ, Turkey
}

Cite this article as: Çay F, Çetinkaya HB, Duran A, Kuloğlu T, Kanat BH, Üstündağ B. The importance of urotensin II level in the diagnosis of acute mesenteric ischemia. Anatolian Curr Med J 2022; 4(1); 59-63.

\begin{abstract}
Aim: This study was conducted to investigate the diagnostic importance of Urotensin II (UT-II) levels, which cause vasodilation as a compensation mechanism in the early phase of Acute Mesenteric Ischemia (AMI). For this purpose, human plasma urotensin was studied for the first time in the literature for the early diagnosis of mesenteric ischemia.

Material and Method: The study consisted of 60 patients. The patients were divided into three groups: Group 1: group with mesenteric ischemia (n: 20); Group 2: group with abdominal pain and with no mesenteric ischemia (n: 20); Group 3: control group with no complaints (n: 20). The blood samples were taken from the patients through peripheral venous access, and Urotensin II (UT-II), Aspartate Aminotransferase (AST), Alkaline phosphatase (ALP), Lactate, and D-dimer levels were measured.

Results: While a significant increase was found between Group1 and Group 2 and between Group 1 and Group 3 in terms of UT-II values ( $\mathrm{p}<0.05$ ), no significant difference was found between Group2 and Group3 ( $>0.05)$. A significant increase was found between Group1 and Group2 and between Group1 and Group3 regarding AST values ( $\mathrm{p}<0.05)$, but there was no significant difference between Group2 and Group3 ( $\mathrm{p}>0.05)$. There was a significant increase between Group 1 and Group3 in terms of $\mathrm{D}$-dimer values $(\mathrm{p}<0.05)$, but no significant difference was seen between Group1 and Group2 and between Group2 and Group3 ( $\mathrm{p}>0.05)$. There was no significant difference between the groups concerning ALP and Lactate values $(\mathrm{P}>0.05)$.

Conclusion: It was concluded that UT-II could be used in the diagnosis of AMI but that there was a need for comprehensive studies investigating the changes in ischemia time-related UT-II serum levels.
\end{abstract}

Keywords: Acute mesenteric ischemia, urotensin-II, D-dimer

\section{INTRODUCTION}

Acute Mesenteric Ischemia (AMI) is a vascular pathology of the gastrointestinal system. It is one of the causes of acute abdomen, which is rarely seen but has very high mortality rates in the community. Early diagnosis is one of the most important factors determining mortality. Despite advances in technology and appropriate diagnosis and treatment, mortality remains high (1). Two main pathophysiological mechanisms can lead to mesenteric ischemia: acute thromboembolic vascular occlusion and non-occlusive mesenteric ischemia (NOMI) $(2,3)$.

Although computed tomography angiography (CTA) gives satisfactory results for the diagnosis of occlusive mesenteric ischemia, the use of CTA is difficult because patients are in intensive care, elderly and immobile. It is very difficult to make a definitive diagnosis of NOMI, which constitutes $20-30 \%$ of AMI cases radiologically (4).

The inadequacy of anamnesis, physical examination, and radiological examinations in making the diagnosis and the high mortality rates of the disease have brought up the search for a specific biomarker.

The splanchnic circulation receives $15-35 \%$ of cardiac output, depending on nutritional status. However, oxygen extraction is relatively low, taking into account the oxygen-carrying capacity of the portal vein to the liver. 
Therefore, blood flow decreases by more than $50 \%$ before the small intestine becomes ischemic (5). In addition, the intestines automatically regulate oxygen availability through enhanced oxygen extraction and perfusion due to early vasodilation. (6) Therefore, we believe that UTII, a vasodilator peptide, increases in the early period of ischemia.

UT-II is an important molecule in the pathophysiology of human diseases. Many studies have observed increased plasma levels of UT-II, which is a potent vasoconstrictor, in the tissues of numerous disease conditions, including hypertension, preeclampsia, atherosclerosis, heart failure, pulmonary hypertension, diabetes, renal failure, and various metabolic syndromes. (7). However, in recent studies, UT-II has been shown to have a vasodilator effect in some veins such as the pulmonary artery and mesenteric arteries. UT-II has been found to be high in patients with low blood pressure in hemodialysis, and this is thought to be due to its vasodilator characteristic (8). Studies on intravenous infusions of UT-II in animals have also shown that UT-II can cause vasodilation (9).

This study was conducted to investigate whether UT--II levels, which may cause vasodilation as a compensatory mechanism in the early period in patients with AMI, could be used as a diagnostic marker. With this study, human Plasma Urotensin was studied for the early diagnosis of mesenteric ischemia for the first time in the literature.

\section{MATERIAL AND METHOD}

The study was initiated with the approval of the FIrat University Non-Interventional Researchs Ethics Committee (Date: 2013, Decision No: 2013/04-06). All procedures were performed adhered to the ethical rules and principles of the Helsinki Declaration.

The study was conducted in the General Surgery and Emergency Medicine Clinic. A detailed anamnesis was taken from the patients included in the study, and their physical examinations were done. Blood samples were taken from the patients through peripheral venous access. The tests were done at the Central Laboratory of Medical Faculty. The serum AST, ALP, lactate, and D-dimer levels were measured. In addition, for UT-II measurement, blood samples of $4 \mathrm{ml}$ placed in an EDTA tube were centrifuged for 4 minutes at $5000 \mathrm{rpm}$. After centrifugation, $1 \mathrm{ml}$ sample was taken from the serum and stored at $-20^{\circ} \mathrm{C}$ until the day when UT-II would be determined. Patient names and numbers were written on each sample and were listed and archived.

\section{Biochemical Examination}

1. Urotensin II: UT-II level was studied after the serums previously taken from the patients and stored at $-20^{\circ} \mathrm{C}$ were thawed. Features of the human urotensin II Elisa kit used in our study: Kit brand- (date): Rel Assay Diagnostic Research and Clinical Chemistry; Assay range: 5 pg/ $\mathrm{mL}-1000 \mathrm{pg} / \mathrm{mL}$; Sensitivity: $2.23 \mathrm{pg} / \mathrm{mL}$; Intra assay: $\mathrm{CV}<10 \%$; inter-assay: $\mathrm{CV}<12 \%$.

2. Serum AST measurement was performed by using the Olympus AU 2700 (Olympus Inc Corporation, Japan) autoanalyzer and original kits.

3. D-dimer measurement was performed by using Siemens CA1500 SYSMEX device (Siemens Healthcare Diagnostic Inc, USA).

4. Serum ALP measurement was performed by using the Olympus AU 2700 (Olympus Inc Corporation, Japan) autoanalyzer and original kits.

5. Lactate plasma levels were determined by using the Siemens Rapidlab-1265 (Siemens Healthcare Diagnostic Inc, USA) device.

\section{Patient Groups}

Group-1 ( $\mathrm{n}=20)$ - Acute Mesenteric Ischemia Group (AMI group): This group consisted of patients who were diagnosed with mesenteric ischemia by laparotomy or those who were diagnosed by CTA as they could not undergo a laparotomy.

Group-2 ( $\mathrm{n}=20)$ - Abdominal Pain Group: This group consisted of patients who presented to the emergency department with acute abdomen pain but did not have ischemia or necrosis due to acute mesenteric vascular occlusion, and had other underlying pathologies.

Group-3 ( $n=20)$ - Control Group: The control group consisted of subjects who did not have a known pathology to cause abdominal pain.

\section{Statictical Analysis}

The data obtained in the study were presented as mean \pm standard deviation values. One-way analysis of variance (ANOVA) tests, post-ANOVA tests, Tukey B, and Scheffe tests were used to evaluate the intergroup differences. In addition, Pearson and Spearman correlation tests were used to examine the correlations between the data in the groups. $\mathrm{p}<0.05$ was accepted as the level of significance.

\section{RESULTS}

The study included a total of 60 patients, including 20 patients who were admitted to the General Surgery Clinic of Frrat University Faculty of Medicine between January 2013 and January 2016 with a diagnosis of AMI (Group-1), 20 patients who had non-specific abdominal pain (Group-2), and 20 randomly selected patients with no abdominal pain (Group-3). There were 14 female and 6 male patients in Group 1 with a mean age of 69.6 
(40-93), 12 female and 8 male patients in Group 2 with a mean age of 61.6 (42-83), and 12 female and 8 male patients in Group 3 with a mean age of 40.6 (23-73) (Table 1).

\begin{tabular}{|lccc|}
\hline \multicolumn{4}{|c|}{ Table 1. Demographic characteristics of the groups } \\
\hline & Female & Male & Mean age (years) \\
\hline Group-1 & 14 & 6 & 69.9 \\
Group-2 & 12 & 8 & 61.6 \\
Group-3 & 12 & 8 & 40.6 \\
\hline
\end{tabular}

The statistical findings for the comparison of the gender and biochemical parameters of the patients in Group 1 indicated that there was no statistically significant difference between gender and UT-II, AST, and ALP values. Lactate and $\mathrm{D}$-dimer values were found to be significantly higher in male patients (Mann-Whitney Test; $\mathrm{p}<0.05)$. There was no statistically significant difference between the age and biochemical parameters of the patients in Group-1 (Summarize Test; p >0.05).

While a significant increase was observed between Group1 and Group2 and also between Group1 and Group3 $(\mathrm{p}<0.05)$ regarding AST values, no significant difference was observed between Group2 and Group3 $(\mathrm{P}>0.05)$. There was a significant increase between Group1 and Group3 in terms of D-dimer values $(\mathrm{p}<0.05)$, but no significant difference was observed between Group1 and Group2 and between Group2 and Group3 $(\mathrm{P}>0.05)$. There was no significant difference between the groups relating to ALP and Lactate values $(\mathrm{P}>0.05)$. All blood values are given in Table 2 .

\begin{tabular}{|lccc|}
\hline \multicolumn{4}{|c|}{ Table 2. Mean values in the groups } \\
\hline & Group-1 & Group-2 & Group-3 \\
\hline AST (U/L) & $76.60 \pm 88.96$ & $27.46 \pm 13.01$ & $26.05 \pm 9.89$ \\
LAKTAT (mEq/L) & $2.5 \pm 1.6$ & $1.8 \pm 0.91$ & $2.9 \pm 1.2$ \\
D-DİMER (ng/mL) & $5.8 \pm 7.6$ & $0.39 \pm 0.28$ & $0.07 \pm 0.03$ \\
ALP (IU/L) & $95.46 \pm 28.66$ & $98.81 \pm 54.91$ & $83.3 \pm 23.2$ \\
\hline
\end{tabular}

Urotensin-II (UT-II) values in 6 patients in group 1 were higher than $1280 \mathrm{pg} / \mathrm{ml}$, which is the highest value that the device can read. The UT-II values of these six patients were included in the study as 1280 $\mathrm{pg} / \mathrm{ml}$. In group 3, UT-II values in two patients were lower than $9,917 \mathrm{pg} / \mathrm{ml}$, which is the lowest value that the device can read. The UT-II values of these two patients were included in the study as $9,917 \mathrm{pg} /$ $\mathrm{ml}$. The mean Urotensin II value was $502.34 \pm 471.57$ $\mathrm{pg} / \mathrm{ml}$ in Group $-1,240.87 \pm 123.98 \mathrm{pg} / \mathrm{ml}$ in Group-2, and $202.48 \pm 58.18 \mathrm{pg} / \mathrm{ml}$ in Group-3. The difference between the UT-II values of Group2 and Group3 was not significant $(\mathrm{P}>0.05)$. When Group2 was compared to Group3 and Group1, a significant increase was

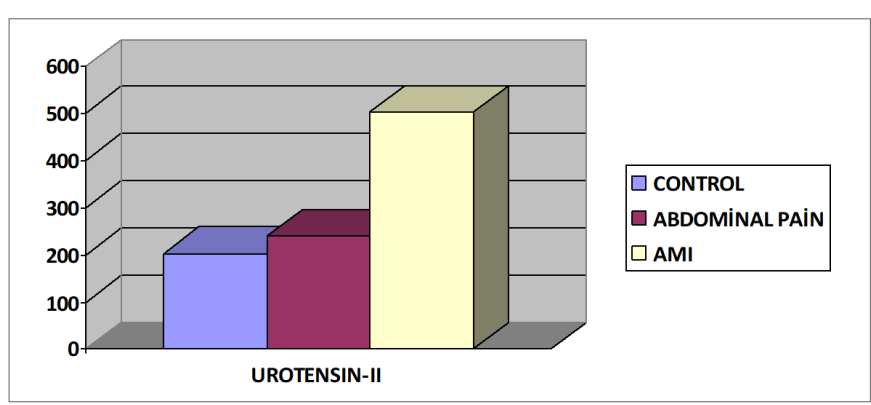

Figure 1. Comparison of UT-II values according to all groups

observed in UT-II values $(\mathrm{p}<0.05)$ (Figure 1).

\section{DISCUSSION}

There is no clear protocol for the diagnosis and treatment of acute mesenteric ischemia yet. The most important factor affecting mortality and morbidity is early diagnosis. Therefore, many studies have been conducted for early diagnosis, but a parameter with early diagnostic efficiency to increase the survival rate has not been found yet (10)

The current diagnostic tool with the highest sensitivity and specificity for the diagnosis of AMI is computed tomography angiography (CTA). Nevertheless, even high-tech diagnostic equipment such as CTA can sometimes miss acute obstructive intestinal ischemia $(11,12)$. In addition, there are also misdiagnosed cases in CTA examinations (13). It may not always be possible to make diagnoses with CTA in non-stable patients hospitalized in the intensive care unit and under mechanical ventilation. At the same time, the sensitivity and specificity of BTA are low in NOMI, which makes up $25 \%$ of all AMI cases (4).

More than $90 \%$ of patients with ischemia have abnormally high leukocyte counts. The second most common abnormal finding is metabolic acidosis with high lactate levels occurring in $88 \%$ of the cases (14). Patients may present with lactic acidosis due to dehydration and decreased oral intake. Therefore, the distinction between early ischemia and irreversible intestinal damage based on lactate level alone is unreliable unless accompanied by other clinical evidence. Elevated serum lactate levels of $>2$ $\mathrm{mmol} / \mathrm{l}$ have been associated with irreversible intestinal ischemia in the diagnosis of acute AMI (15). Kulaçoğlu et al. (16) found that there was a significant increase in plasma lactate levels in rat models of mesenteric ischemia. Lange et al. (17) found lactate $100 \%$ sensitive but $42 \%$ specific as a biochemical marker for AMI. Acosta et al. (18) concluded that plasma lactate levels would not be recommended as a diagnostic marker in suspected intestinal ischemia in the experimental AMI they created. In another study, it was concluded that serum lactate measurement was not a specific confirmatory factor for diagnosis (19). As in similar studies, no statistically 
significant difference was observed between the groups relating to lactate values in our study.

$\mathrm{D}$-dimer is a degradation product specific to fibrin clots, with a plasma half-life of 4-8 hours. There are studies on dynamic D-dimer levels in AMI (20-22). D-dimer has been reported to be an independent risk factor for intestinal ischemia, reflecting continued clot formation and endogenous degradation via fibrinolysis (23). Kurt et al. (24) found the sensitivity of D-dimer in AMI as $88.8 \%$, specificity as $90 \%$, positive predictive value (PPV) as $88.8 \%$, and negative predictive value (NPV) as $100 \%$ in an experimental study and concluded that D-dimer may be practical in the early diagnosis of acute mesenteric ischemia. Acosta et al. (25) showed that D-dimer was significantly higher in patients with AMI than in patients with inflammatory bowel disease and intestinal obstruction. The sensitivity of the $\mathrm{D}$-dimer value has been reported as $100 \%$ and the specificity as $36 \%$. Cudnik et al. (26) reviewed data from five studies evaluating D-dimer as a biomarker for AMI, reporting a sensitivity of $96 \%$ and a much lower specificity of $40 \%$. In a small series of 10 patients with confirmed AMI, Block et al. (27) noted that $\mathrm{D}$-dimer was positive in all individuals. In our study, we found that $\mathrm{D}$-dimer was significant in the diagnosis of AMI.

As a result of acute mesenteric ischemia, necrosis and ultimately bacterial translocation-related sepsis occur. Septic complications contribute to multiple organ damage. An increase in liver function tests can be observed with liver damage. Diebel et al. (28) observed an increase in liver function tests with liver injury by decreasing portal blood flow. In our study, there was a statistically significant increase in serum AST levels in Group1. These results of ours are in parallel with the findings of Diebel et al.

Urotensin II peptide has been isolated in vascular endothelium, heart, leukocytes, liver, adrenal glands, pituitary, brain, spinal cord, kidney, spleen, leukocytes, small intestine, colon, placenta, and other tissues (29). Stirrat et al. (30) demonstrated that UT-II caused vasodilation in human mesenteric and pulmonary arteries through its receptors on the endothelium. Similarly, Katano et al. (31) showed that the vasodilator effects of UT-II might be dependent on endothelial dysfunction and suggested that this molecule exerted its vasodilatory effects through $\mathrm{NO}$ and prostaglandin formation. In our study, UT-II levels in the patients in the AMI group were found to be significantly higher than the patients in the other groups.

Our study has some limitations. For example, the number of patients in our study was low, and we could not measure the stage of ischemia in our patients in group 1 when they presented to our clinic.
The strength of our study is that it is a biomarker study on human serum studied for the first time in the literature.

We have concluded that UT-II can be used in the biochemical diagnosis of AMI. However, there is a need for more studies to investigate the ischemia time-related changes in UT-II serum levels.

\section{ETHICAL DECLARATION}

Ethics Committee Approval: The study was initiated with the approval of the Firat University Non-Interventional Researchs Ethics Committee (Date: 2013, Decision No: 2013/04-06).

Informed Consent: All patients signed the free and informed consent form.

Referee Evaluation Process: Externally peer-reviewed.

Conflict of Interest Statement: The authors have no conflicts of interest to declare.

Financial Disclosure: The authors declared that this study has received no financial support.

Author Contributions: All of the authors declare that they have all participated in the design, execution, and analysis of the paper, and that they have approved the final version.

Ackowledgements: All authors would like to thank Ziya Çetinkaya, who is not alive at the moment and who contributed a lot to this study.

\section{REFERENCES}

1. Acosta-Mérida MA, Marchena-Gómez J, Saavedra-Santana P, Silvestre-Rodríguez J, Artiles-Armas M, Callejón-Cara MM. Surgical outcomes in acute mesenteric ischemia: has anything changed over the years? World J Surg 2020; 44: 100-7.

2. Van Noord D, Kolkman JJ. Functional testing in the diagnosis of chronic mesenteric ischemia. Best Pract Res Clin Gastroenterol 2017; 31: 59-68.

3. Bourcier S, Oudjit A, Goudard G, et al. Diagnosis of nonocclusive acute mesenteric ischemia in the intensive care unit. Ann Intensive Care 2016; 6: 112.

4. Clair DG, Beach JM. Mesenteric ischemia. N Engl J Med 2016; 374: 959-68.

5. Rosenblum JD, Boyle CM, Schwartz LB. The mesenteric circulation. Anatomy and physiology. Surg Clin North Am 1997; 77: 289-306.

6. Haglund U, Bergqvist D. Intestinal ischemia-the basics. Langenbeck's Arch Surg 1999; 384: 233-8.

7. Ross B, McKendy K, Giaid A. Role of urotensin II in health and disease. Am J Physiol 2010; 298: 1156-72.

8. Mosenkis A, Kallem RR, Danoff TM, Aiyar N, Bazeley J, Townsend RR. Renal impairment, hypertension and plasma urotensin II. Nephrology Dialysis Transplantation 2011; 26: 609-14.

9. Gardiner SM, March JE, Kemp PA, Davenport AP, Bennett T. Depressor and regionally-selective vasodilator effects of human and rat urotensin II in conscious rats. Br J Pharmacol 2001; 132: 1625-9.

10. Bobadilla JL. Mesenteric ischemia. Surg Clin North Am 2013; 93: 925-40. 
11. Bourcier S, Oudjit A, Goudard G, et al. Diagnosis of nonocclusive acute mesenteric ischemia in the intensive care unit. Ann Intensive Care 2016; 6: 112.

12. Kärkkäinen JM, Acosta S. Acute mesenteric ischemia (part I) Incidence, etiologies, and how to improve early diagnosis. Best Pract Res Clin Gastroenterol 2017; 31: 15-25.

13. Kozuch PL, Brandt LJ. Review article: diagnosis and management of mesenteric ischaemia with an emphasis on pharmacotherapy. Aliment Pharmacol Ther 2005; 21: 201-15.

14. Kougias P, Lau D, El Sayed HF, Zhou W, Huynh TT, Lin PH. Determinants ofmortality and treatment outcome following surgical interventions for acutemesenteric ischemia. J Vasc Surg 2007; 46: 467-74.

15. Nuzzo A, Maggiori L, Ronot $M$, et al. Predictive factors of intestinal necrosis in acute mesenteric ischemia: prospective study from an intestinal stroke center. Am J Gastroenterol 2017; 112: 597-605.

16. Kulaçoğlu H, Kocaerkek Z, Moran M, Kulah B, Atay Ç, Kulaçoğlu S. Diagnostic value of blood D-dimer level in acute mesenteric ischaemia in the rat: An experimental study. Asian J Surg 2005; 28: $131-5$

17. Lange $H$, Jackel R. Usefulness of plasma lactate concentration in the diagnosis of acute abdominal disease. Eur J Surg 1994; 160: 381-384.

18. Acosta S, Nilsson TK, Malina J, Malina M. L-lactate after embolization of the superior mesenteric artery. J Surg Res 2007; 143: 320-8.

19. Meyer T, Klein P, Schweiger H, Lang W. How can the prognosis of acute mesenteric artery ischemia be improved? Results of a retrospektive analysis. Zentralbl Chir Nürnberg 1998; 123: 230-4.

20. Fernández-Ruiz I. Biomarkers: extended predictive value of d-dimer. Nat Rev Cardiol 2018; 15: 198.

21. Yang $\mathrm{K}$, Wang $\mathrm{W}$, Zhang $\mathrm{WH}$, et al. The combination of $\mathrm{D}$-dimer and peritoneal irritation signs as a potential indicator to exclude the diagnosis of intestinal necrosis. Medicine 2015; 94: e1564.

22. Montagnana M, Danese E, Lippi G. Biochemical markers of acute intestinal ischemia: possibilities and limitations. Ann Transl Med 2018; 6: 341.

23. Powell A, Armstrong P. Plasma biomarkers for early diagnosis of acuteintestinal ischemia. Semin Vasc Surg 2014; 27: 170-5.

24. Kurt Y, Akin ML, Demirbas S, et al. D-dimer in early diagnosis of acute mesenteric ischemia secondary to arterial occlusion in rats. Eur Surg Res 2005; 37: 216-9.

25. Kanda T, Fujii H, Tani T. Intestinal fatty acid-binding protein is a useful diagnostic marker for mesenteric infarction in humans. Gastroenterology 1996; 110: 339-43.

26. Cudnik MT, Darbha S, Jones J, Macedo J, Stockton SW, Hiestand BC. The diagnosis of acute mesenteric ischemia: A systematic review and meta-analysis. Acad Emerg Med 2013; 20: 1087-100.

27. Block T, Nilsson TK, Björck M, Acosta S. Diagnostic accuracy of plasma biomarkers for intestinal ischaemia. Scand J Clin Lab Invest 2008; 68: 242-8.

28. Diebel LN, Wilson RF, Dulchavsky SA, Saxe J. Effect of increased intraabdominal pressure on hepatic arterial, portal venous, and hepatic microcirculatory blood flow. J Trauma 1992; 33: 279-82.

29. Acosta S, Nilsson TK, Bjorck M. D-dimer testing in patients with suspected acute thromboembolic occlusion of the superior mesenteric artery. Br J Surg 2004; 91: 991-4.

30. Stirrat A, Gallagher M, Douglas SA. Potent vasodilator responses to human urotensin-II in human pulmonary and abdominal resistance arteries. Am J Physiol Heart Circ Physiol 2001; 280: 925-8.

31. Katano Y, Ishihata A, Aita T, Ogaki T, Horie T. Vasodilator effect of urotensin II, one of the most potent vasoconstricting factors, on rat coronary arteries. Eur J Pharmacol 2000; 402: 5-7. 